\title{
aportación
}

\section{en torno a la valoración de la calidad resistente del hormigón \\ de estructuras por medio de probetas testigo extraídas por corte}

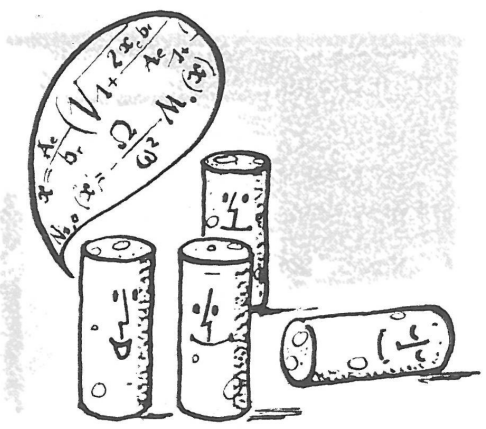

J. L. RAMIREZ ORTIZ, Prof. Dr. ingeniero industrial

J. M. BARCENA DIAZ, ingeniero industrial

sinopsis se estudian en este trabajo de investigación experimental dos problemas relacionados con el control de obra de hormigón endurecido:

1. El del deterioro que experimenta la resistencia del testigo de hormigón debido al corte necesario diámetros de los testigos.

2. Verificar la pérdida de resistencia de elementos a los que se ha perforado, para la extracción de un testigo cilíndrico, procediendo después al relleno del agujero resultante.

\section{introducción}

El objeto del presente trabajo es presentar unos primeros resultados de la investigación experimental emprendida por los autores " en torno a problemas que se suscitan en la inspección de obras de hormigón ya endurecido, cuando se descubren elementos estructurales de baja calidad.

Evidentemente son muchos y variados los problemas que en este campo se prestan a estudio y ensayo, y es nuestro propósito seguir experimentando en dicha dirección; pero en esta ocasión nos limitaremos a aportar algunos resultados obtenidos en torno a la valoración de la calidad resistente del hormigón de estructuras por medio de probetas testigos extraídas por corte.

En el control de obra terminada de hormigón el proceso que generalmente seguimos es el siguiente:

Inspección esclerométrica de pilares en dos niveles, y de vigas en el centro de la luz y en las dos cabezas de pilar hormigonadas a la vez que la viga.

Con ello queda conocida la calidad de los pilares en tres alturas, y de las vigas en el centro del vano y empotramientos.

\footnotetext{
- Los trabajos de investigación se han realizado merced a la Ayuda a la Investigación concedida por el Ministerio de Educación y Ciencia a los autores, en el marco de la Cátedra de Estructuras de la Escuela Superior de Ingenieros Industriales de Bilbao, de la que J. L. Ramírez Ortiz es titular.

Los ensayos se han realizado en los Laboratorios de la Cátedra de Estructuras y en los Laboratorios de Ensayos e Investigación Industrial aL. J. Torróntegui a anexos a dicha Escuela.
} 
Clasificación de los distintos elementos de la estructura, vigas y pilares, en varios grupos, por su valor esclerométrico (media de diez rebotes) mínimo o por el valor que mejor les caracterice, de los tres que se han obtenido de acuerdo con el apartado anterior.

Extracción de testigos cilíndricos de hormigón, generalmente de $10 \mathrm{~cm}$ de diámetro, de diversos elementos de cada uno de los niveles esclerométricos en que se han clasificado los elementos estructurales, y rotura de dichos testigos debidamente preparados.

Determinación de una curva de correlación, con un grado de confianza del $95 \%$, entre nivel de rebote y resistencia real del testigo extraído.

Con esto último la dirección de la obra tiene datos suficientes para saber qué elementos hay que sustituir o reparar y cuáles pueden quedar con suficiente coeficiente de seguridad.

Entre los numerosos problemas, como decíamos antes, que se plantean a lo largo de este proceso, ha habido dos que hemos querido tratar de estudiar con mayor interés:

El primero de ellos es el motivado porque el corte del testigo con la corona de diamante supone físicamente un deterioro de su superficie, variable con la relación de tamaño máximo de árido a diámetro de corona cortante y con la misma calidad del hormigón que se quiere poner de manifiesto.

Al ensayar el testigo, parece claro que su resistencia deberá ser algo menor que si no hubiera sufrido deterioro y, por lo tanto, que perjudica al elemento estructural que representa.

El objeto, pues, de la primera parte del trabajo ha sido el poner de manifiesto las diferencias de resistencia obtenidas sobre un mismo hormigón mediante probetas testigo de varios diámetros extraídas por corte, de probetas moldeadas en el transcurso del hormigonado, y de los mismos pilares de tamaño natural, haciendo una estimación del deterioro producido por el corte de los testigos. Algunos investigadores (1, 2, 3 y 4) han trabajado sobre estos temas con resultados de gran interés, pero no han dado valores para este último efecto.

El segundo problema sobre el que se ha querido aportar datos, del que no conocemos referencias de trabajos similares, ha sido el de conocer cómo queda, desde el punto de vista resistente, un pilar, al que después de extraerle una probeta cilíndrica de $10 \mathrm{~cm}$ de diámetro y constatar que su resistencia es suficientemente buena, se le rellena por diferentes métodos el agujero provocado para la extracción del testigo.

El contenido de las páginas que siguen constituye una primera fase de resultados, debido a que en ella no se ha llegado a rotura de pilares a tamaño natural. Dicha parte del programa está actualmente en ejecución, pero se ha pensado que los datos obtenidos hasta aquí tienen suficiente interés para ser considerados separadamente.

\section{Influencia del corte de los testigos}

\subsection{PLANTEAMIENTO DE LA INVESTIGACION}

Los aspectos que se han querido tocar en esta primera parte del trabajo han sido los siguientes:

a) Deterioro del testigo de hormigón por el corte producido para su extracción.

b) Influencia en el mismo de diferentes relaciones de diámetro de testigo a tamaño máximo de árido. 
c) Relación entre resistencias de los testigos de diferentes diámetros y grado de confianza de las mismas.

d) Diferencias entre resistencias de probetas moldeadas en el transcurso de la obra y las extraídas por corte.

e) Influencia de la dirección de la extracción de la probeta testigo en relación con la de hormigonado.

f) Influencia, en los apartados anteriores, del nivel de resistencia del hormigón de que se trate.

Los puntos anteriores han orientado el planteamiento de los ensayos que se explica a continuación.

Así, para poner de manifiesto la influencia de la calidad del hormigón se han repetido todos los ensayos en hormigones de resistencias nominales a 90 días de 90,130 y $200 \mathrm{kp} / \mathrm{cm}^{2}$.

EI efecto de la relación entre tamaño máximo de árido y diámetro de corona se ha tenido en cuenta utilizando un tamaño máximo de árido de $3 \mathrm{~cm}$ y coronas cortantes de 5,10 y $15 \mathrm{~cm}$ de diámetro.

Se ha comparado también el efecto del hormigonado del elemento en sentido vertical u horizontal, preparando dos piezas idénticas alargadas, hormigonando cada una en un sentido.

El conjunto de resultados que se ha buscado obtener de cada masa para su comparación y contestación a las cuestiones antes planteadas ha sido el siguiente:

Resistencias de probetas moldeadas cilíndricas de $15 \times 30 \mathrm{~cm}$.

Resistencias de probetas extraídas por corte con corona;

- de 5, 10 y $15 \mathrm{~cm}$ de diámetro y doble de altura;

- de piezas hormigonadas en horizontal y vertical.

Resistencias de probetas moldeadas prismáticas de $20 \times 20 \times 50 \mathrm{~cm}$.

El proceso de preparación de probetas se ha desarrollado como sigue:

De cada uno de los tres niveles de calidad del hormigón se han realizado cinco masas, con cada una de las cuales se han moldeado seis probetas cilíndricas de $15 \times 30 \mathrm{~cm}$, dos prismas de $20 \times 20 \times 50 \mathrm{~cm}$ y dos prismas de $110 \mathrm{~cm}$ de longitud y sección en L, de acuerdo con la figura 1, y de los que posteriormente, a los 85 días, se extraían de cada uno, por corte, seis testigos cilíndricos de cada uno de los diámetros 5,10 y $15 \mathrm{~cm}$, aproximadamente.

Por tanto, después de la rotura a la edad de 90 días, hemos dispuesto para cada nivel de resistencia de 30 valores de todos los tipos de probetas, excepto de los prismas de $20 \times 20 \times$ $\times 50 \mathrm{~cm}$, de los que sólo había 10. La cifra de al menos 30 valores se ha buscado expresamente para dar mayor valor a los análisis estadísticos que posteriormente se han efectuado con los resultados *.

\footnotetext{
* Los análisis estadísticos han sido realizados por D. Pedro Mendizábal Larumbe, Dr. Ingeniero Industrial, Presidente del Comité de Honor de la Asociación Nacional para el Control de la Calidad, a quien agradecemos su valiosa colaboración.
} 


\subsection{DATOS TECNICOS CORRESPONDIENTES A LOS ENSAYOS}

\subsubsection{Aridos}

Se han utilizado áridos de machaqueo calizos de cantera situada dentro del casco urbano de Bilbao. El tamaño máximo ha sido de $30 \mathrm{~mm}$, habiéndose empleado tres tipos: guijo, guijillo y arena.

El contenido en finos en la arena, determinado de acuerdo con la norma UNE 7135 , fue del $14 \%$, valor muy alto, sobre todo si se compara con el máximo del $5 \%$ admitido por la Instrucción Española, pero bastante corriente en la mayor parte de las canteras de caliza de la región. Por ello, para asemejarnos lo más posible a las condiciones de obra, se ha utilizado dicha arena corriente sin lavar o ciclonar.

Las densidades aparentes han sido:

$$
\begin{array}{llllllllll}
- \text { guijo } & \ldots & \ldots & \ldots & \ldots & \ldots & \ldots & \ldots & \ldots & 1.400 \mathrm{~kg} / \mathrm{m}^{3} \\
- \text { guijillo } & \ldots & \ldots & \ldots & \ldots & \ldots & \ldots & \ldots & 1.430 \mathrm{~kg} / \mathrm{m}^{3} \\
-\operatorname{arena} & \ldots & \ldots & \ldots & \ldots & \ldots & \ldots & \ldots & \ldots & \\
\end{array}
$$

La mezcla óptima se ha realizado con el criterio de ajustarla lo más posible a la fórmula de Fuller.

Las proporciones, en peso, de la mezcla óptima han resultado ser:

\begin{tabular}{|c|c|c|c|}
\hline CARACTERISTICAS & $\begin{array}{l}\text { Masa de } \\
90 \mathrm{kp} / \mathrm{cm}^{2}\end{array}$ & $\begin{array}{l}\text { Masa de } \\
130 \mathrm{kp} / \mathrm{cm}^{2}\end{array}$ & $\begin{array}{l}\text { Masa do } \\
200 \mathrm{kp} / \mathrm{cm}^{2}\end{array}$ \\
\hline Guijo $(\%)$ & 50 & 50 & 50 \\
\hline Guijillo (\%) & 10 & 10 & 10 \\
\hline Arena $(\%)$ & 40 & 40 & 40 \\
\hline Cemento P-350 $\left(\mathrm{kg} / \mathrm{m}^{3}\right)$ & 200 & 200 & 270 \\
\hline$A / C$ & 1,30 & 1,08 & 0,80 \\
\hline Cono de Abrams $(\mathrm{cm})$ & 17 & 5 & 5 \\
\hline Docilidad V.B. (s) & - & 3 & 3 \\
\hline Aspecto & Muy fluida & Plástica & Plástica \\
\hline
\end{tabular}

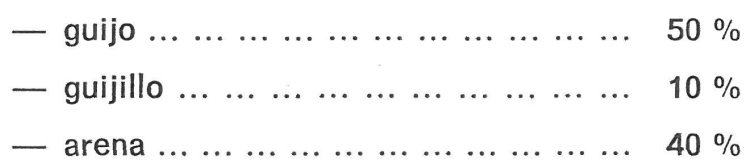

1.2.2. Características de las masas

\subsubsection{Confección y conservación de probetas y testigos}

El hormigón de las masas de 90 y $130 \mathrm{kp} / \mathrm{cm}^{2}$ ha sido compactado a barra. El de la de 200 $\mathrm{kp} / \mathrm{cm}^{2}$ ha sido vibrado. 
Todas las probetas, bloques y testigos cortados de la misma masa se han almacenado en total proximidad, siendo las condiciones ambientales de $20^{\circ} \pm 1^{\circ} \mathrm{C}$ de temperatura y $60-70 \%$ de humedad relativa (fotos 1 y 2 ).

Los ensayos se han efectuado a la edad de 90 días.
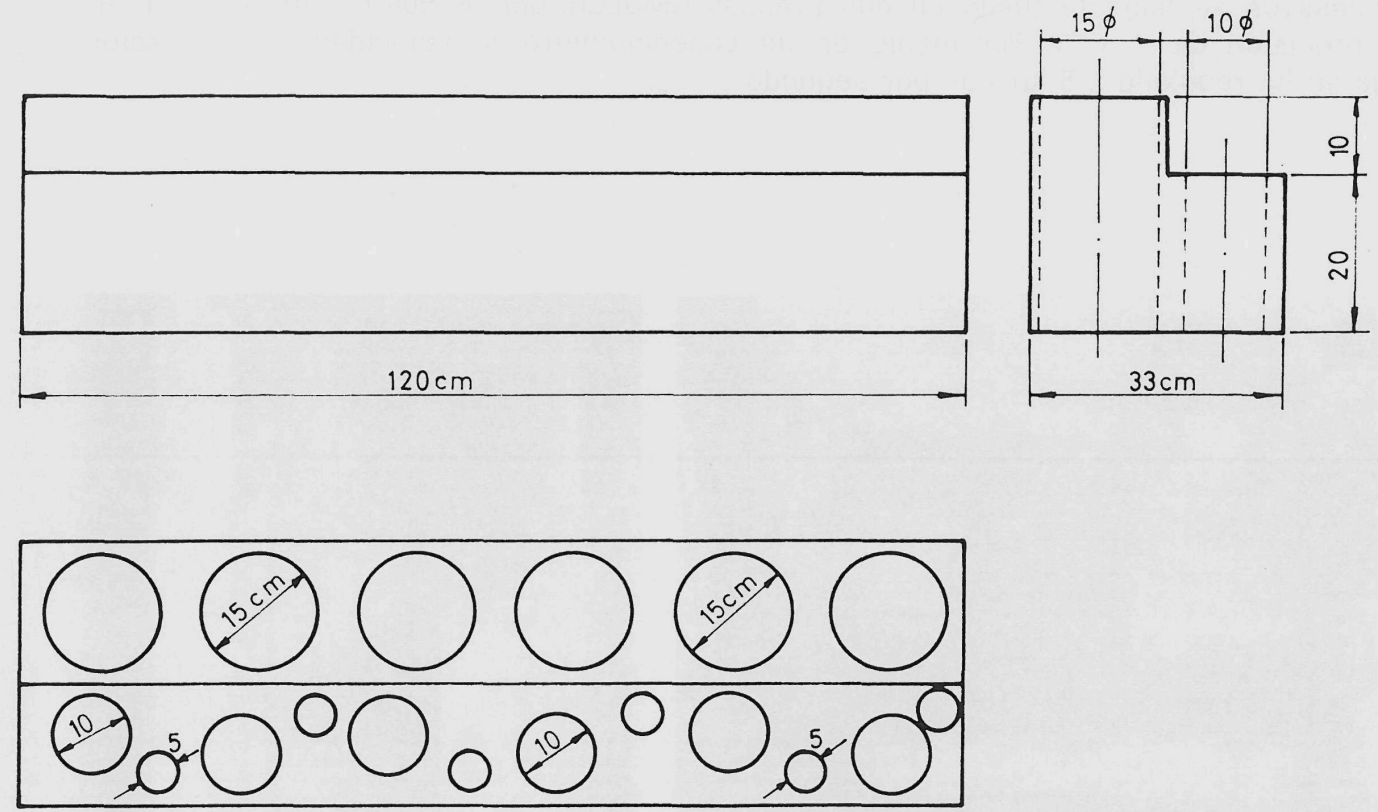

1 Bloque hormigonado horizontal.

1 Bloque hormigonado verticalmente.
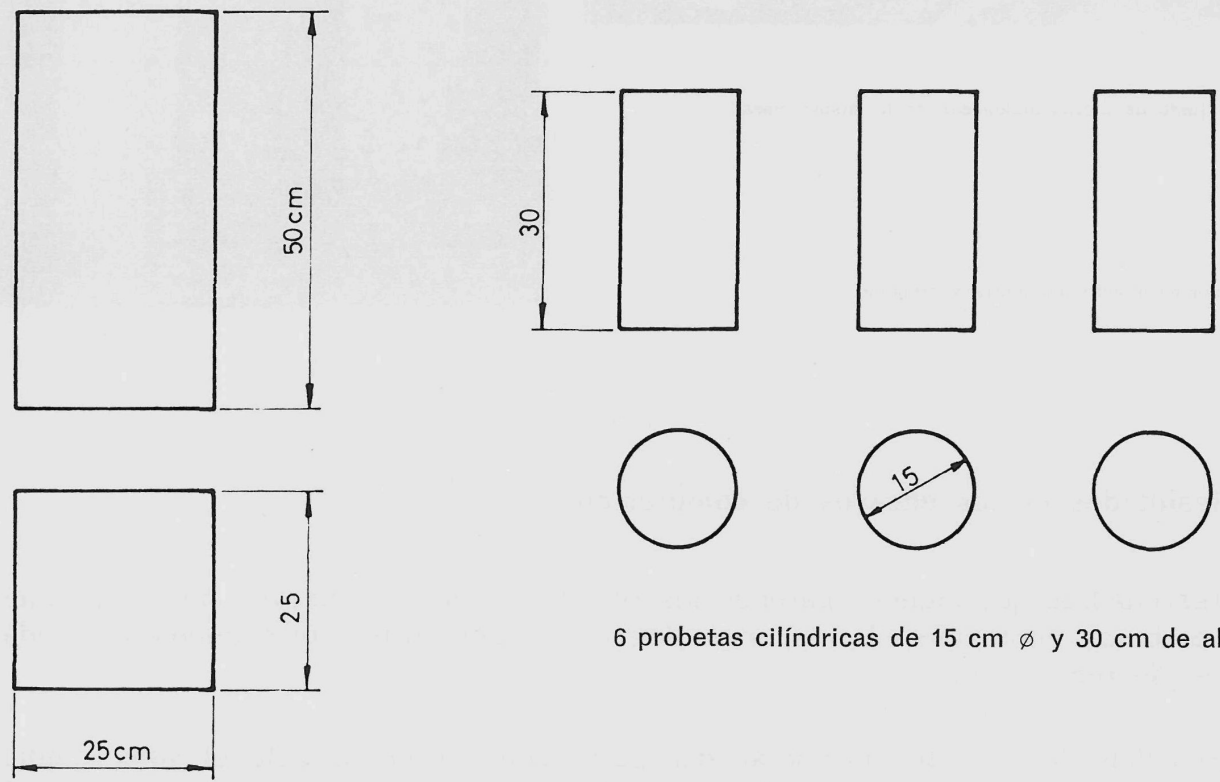

6 probetas cilíndricas de $15 \mathrm{~cm} \varnothing$ y $30 \mathrm{~cm}$ de altura.

2 Bloques hormigonados verticalmente.

Fig. 1. Conjunto de piezas preparadas, del mismo lote de hormigón 
La extracción de probetas por corte se ha hecho con un aparato rotativo cuyo eje gira en carga a 730 r.p.m., siendo las coronas cortantes del tipo de las de polvo de diamante. Dicha extracción se hacía a la edad de 85 días, para una vez refrentadas las caras de contacto con la prensa con mortero de cemento estar listas para ensayo a la edad de 90 días, como ya se ha dicho.

Los ensayos se han efectuado en dos prensas AMSLER, una de $500 \mathrm{t}$ y otra de $50 \mathrm{t}$, ambas de una precisión de $\pm 1 \%$. Por medio de un cadenciómetro la velocidad de aplicación de la carga se ha regulado a $5 \mathrm{kp} / \mathrm{cm}^{2}$ por segundo.

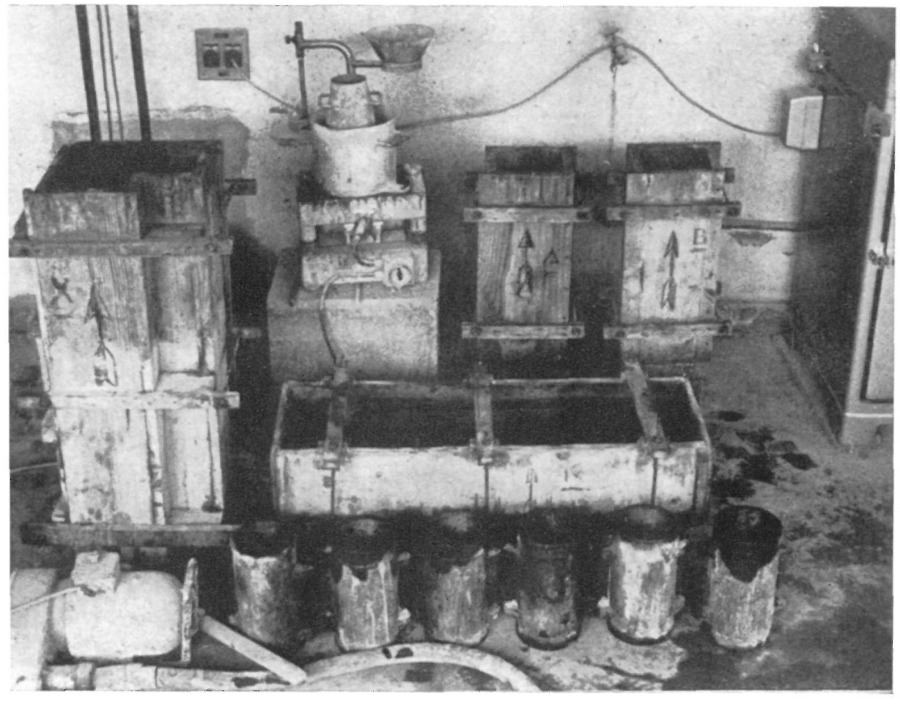

Foto 1. Conjunto de piezas moldeadas de la misma masa.

Foto 2. Almacenamiento de piezas y probetas.

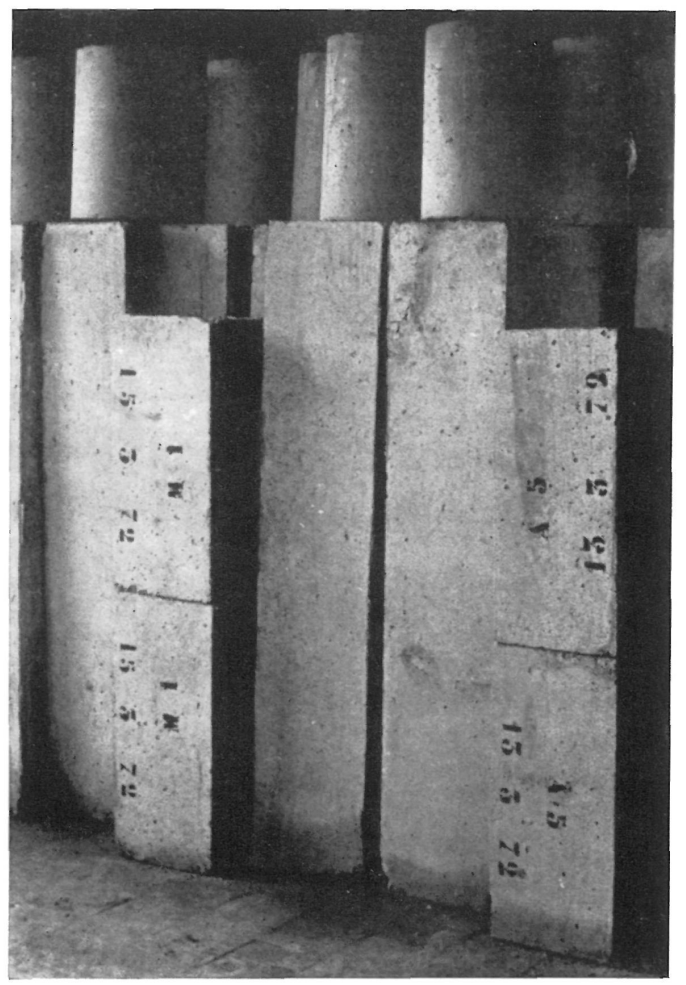

\subsubsection{Resultados de los ensayos de compresión}

En los tres cuadros que siguen aparecen los resultados de los ensayos de compresión de todas las probetas confeccionadas, sean moldeadas o por corte, correspondiendo cada cuadro a un nivel de resistencia.

En las tres filas de la parte inferior se dan para cada tipo de probeta el valor medio de los resultados, su coeficiente de variación y la relación de las medias con la de las probetas cilíndricas moldeadas de acuerdo con la Instrucción Española (cuadros 1, 2 y 3). 


\subsection{CONCLUSIONES}

1. La dispersión en los resultados de los ensayos a compresión de los testigos extraídos por corte aumenta a medida que su diámetro disminuye.

El coeficiente de variación de los resultados de los testigos de 15 y $10 \mathrm{~cm}$ de diámetro está comprendido entre el 5 y el $10 \%$, valores realmente de laboratorio en el campo del ensayo de hormigones. Además, dicho intervalo de valores es el que se presenta también en los resul-

MASA DE $90 \mathrm{kp} / \mathrm{cm}^{2}$

Resístencia a la compresión $\left(\mathrm{kp} / \mathrm{cm}^{2}\right)$

CUADRO

\begin{tabular}{|c|c|c|c|c|c|c|c|c|c|}
\hline \multirow[t]{2}{*}{$\begin{array}{c}\text { Amasada } \\
n\end{array}$} & \multirow[t]{2}{*}{$\begin{array}{l}\text { Probeta } \\
\text { no }\end{array}$} & \multirow{2}{*}{$\begin{array}{l}\text { Mioldeadas } \\
\text { cilindros } \\
\phi=15 \mathrm{~cm} \\
h=30 \mathrm{~cm}\end{array}$} & \multicolumn{2}{|c|}{$\begin{array}{l}\text { Cortadas } \\
\phi=15 \mathrm{~cm} \\
h=30 \mathrm{~cm}\end{array}$} & \multicolumn{2}{|c|}{$\begin{array}{l}\text { Cortadas } \\
\phi=10 \mathrm{~cm} \\
h=20 \mathrm{~cm}\end{array}$} & \multicolumn{2}{|c|}{$\begin{array}{l}\text { Cortadas } \\
\phi=5 \mathrm{~cm} \\
h=10 \mathrm{~cm}\end{array}$} & \multirow{2}{*}{$\begin{array}{l}\text { Moldeadas } \\
\text { prismas } \\
\square 20 \times 20 \mathrm{~cm} \\
h=50 \mathrm{~cm} .\end{array}$} \\
\hline & & & Hor. & vert. & Hor. & vert. & Hor. & Vert. & \\
\hline & & I & II & I I I & IV & v & VI & VII & VIII \\
\hline 1 & $\begin{array}{l}1 \\
2 \\
3 \\
4 \\
5 \\
6\end{array}$ & $\begin{array}{r}96,5 \\
102,5 \\
99,5 \\
102,5 \\
102,5 \\
107,0\end{array}$ & $\begin{array}{l}105,5 \\
108,5 \\
105,5 \\
110,0 \\
107,5 \\
105,6\end{array}$ & $\begin{array}{r}103,5 \\
98,0 \\
103,5 \\
101,5 \\
98,5 \\
95,0\end{array}$ & $\begin{array}{l}119,0 \\
113,5 \\
126,0 \\
109,0 \\
106,0 \\
114,5\end{array}$ & $\begin{array}{l}108,5 \\
106,0 \\
104,5 \\
107,5 \\
101,5 \\
104,5\end{array}$ & $\begin{array}{r}116,5 \\
104,5 \\
97,5 \\
82,0 \\
100,0 \\
103,0\end{array}$ & $\begin{array}{r}91,0 \\
85,0 \\
106,5 \\
98,0 \\
100,0 \\
99,5\end{array}$ & $\begin{array}{l}108,0 \\
107,5\end{array}$ \\
\hline 2 & $\begin{array}{r}7 \\
8 \\
9 \\
10 \\
11 \\
12\end{array}$ & $\begin{array}{r}102,0 \\
102,5 \\
95,0 \\
107,0 \\
103,5 \\
105,5\end{array}$ & $\begin{array}{r}109,5 \\
100,3 \\
105,0 \\
108,0 \\
102,5 \\
99,7\end{array}$ & $\begin{array}{l}104,5 \\
110,0 \\
111,0 \\
115,8 \\
108,5 \\
109,8\end{array}$ & $\begin{array}{l}108,5 \\
103,0 \\
117,5 \\
107,0 \\
106,2 \\
105,5\end{array}$ & $\begin{array}{l}114,5 \\
116,2 \\
107,0 \\
118,0 \\
120,0 \\
122,5\end{array}$ & $\begin{array}{r}130,0 \\
121,0 \\
78,5 \\
93,6 \\
73,7 \\
105,0\end{array}$ & $\begin{array}{r}123,5 \\
83,7 \\
114,5 \\
95,0 \\
105,5 \\
110,0\end{array}$ & $\begin{array}{l}111,5 \\
104,0\end{array}$ \\
\hline 3 & $\begin{array}{l}13 \\
14 \\
15 \\
16 \\
17 \\
18 \\
\end{array}$ & $\begin{array}{l}97,5 \\
95,7 \\
87,8 \\
89,4 \\
98,0 \\
90,5 \\
\end{array}$ & $\begin{array}{l}111,0 \\
115,0 \\
115,0 \\
113,5 \\
118,0 \\
116,0\end{array}$ & $\begin{array}{l}125,0 \\
113,0 \\
120,5 \\
116,5 \\
115,0 \\
125,0\end{array}$ & $\begin{array}{l}120,0 \\
111,0 \\
116,0 \\
122,0 \\
108,0 \\
104,5\end{array}$ & $\begin{array}{r}115,0 \\
112,0 \\
107,0 \\
108,5 \\
95,5 \\
94,3\end{array}$ & $\begin{array}{r}86,5 \\
107,0 \\
107,5 \\
110,5 \\
115,5 \\
103,0\end{array}$ & $\begin{array}{r}92,4 \\
73,0 \\
119,0 \\
92,3 \\
85,3 \\
72,5\end{array}$ & $\begin{array}{l}114,2 \\
105,7\end{array}$ \\
\hline 4 & $\begin{array}{l}19 \\
20 \\
21 \\
22 \\
23 \\
24 \\
\end{array}$ & $\begin{array}{l}106,5 \\
107,0 \\
102,5 \\
119,5 \\
121,0 \\
126,5\end{array}$ & $\begin{array}{l}115,5 \\
114,0 \\
115,0 \\
115,0 \\
106,0 \\
111,5\end{array}$ & $\begin{array}{r}102,5 \\
118,0 \\
114,0 \\
99,0 \\
112,0 \\
120,5\end{array}$ & $\begin{array}{l}137,0 \\
123,5 \\
140,0 \\
132,0 \\
126,0 \\
125,0\end{array}$ & $\begin{array}{r}110,5 \\
109,0 \\
124,5 \\
107,5 \\
108,0 \\
96,3\end{array}$ & $\begin{array}{l}152,0 \\
109,0 \\
129,5 \\
157,5 \\
131,5 \\
105,0\end{array}$ & $\begin{array}{r}99,5 \\
108,5 \\
93,5 \\
103,0 \\
120,5 \\
107,0\end{array}$ & $\begin{array}{l}107,5 \\
107,7\end{array}$ \\
\hline 5 & $\begin{array}{l}25 \\
26 \\
27 \\
28 \\
29 \\
30 \\
\end{array}$ & $\begin{array}{r}102,0 \\
96,2 \\
97,3 \\
96,2 \\
96,2 \\
91,2 \\
\end{array}$ & $\begin{array}{r}105,5 \\
97,4 \\
104,5 \\
105,0 \\
114,5 \\
707,0\end{array}$ & $\begin{array}{r}120,5 \\
108,5 \\
119,0 \\
118,0 \\
102,5 \\
99,5\end{array}$ & $\begin{array}{r}124,0 \\
120,5 \\
106,0 \\
93,5 \\
120,5 \\
96,0\end{array}$ & $\begin{array}{r}116,5 \\
117,0 \\
118,5 \\
107,5 \\
95,3 \\
82,5\end{array}$ & $\begin{array}{r}98,8 \\
91,7 \\
106,5 \\
134,0 \\
113,5 \\
82,7\end{array}$ & $\begin{array}{r}125,0 \\
106,0 \\
75,0 \\
110,0 \\
102,5 \\
73,7\end{array}$ & $\begin{array}{l}91,2 \\
93,2\end{array}$ \\
\hline & $\begin{array}{l}\text { Valor } \\
\text { medio }\end{array}$ & 101,6 & 108,9 & 110,0 & 115,4 & 108,5 & 108,2 & 99,0 & 105,0 \\
\hline & $\begin{array}{c}\text { Coef. } \\
\text { variación \% }\end{array}$ & 8,7 & 6,1 & 10,1 & 9,6 & 8,5 & 18,4 & 15,0 & 7,0 \\
\hline & Res./I & 1 & 1,07 & 1,08 & 1,14 & 1,07 & 1,06 & 0,97 & 1.030 \\
\hline
\end{tabular}


tados de las probetas cilíndricas moldeadas según Normas de $15 \mathrm{~cm}$ de diámetro por $30 \mathrm{~cm}$ de altura.

De ello parece deducirse una alta fiabilidad en los resultados obtenidos en las probetas de 15 y $10 \mathrm{~cm}$ de diámetro extraídas por trépano, a las que, en cuanto a dispersión, no parece que el efecto físico del corte cause un aumento detectable.

El coeficiente de variación en los testigos de $5 \mathrm{~cm}$ de diámetro ya es bastante mayor, entre 15 y $30 \%$, lo que ya hace menos confiables los resultados. Ello será debido al deterioro por

MASA DE $130 \mathrm{kp} / \mathrm{cm}^{2}$ Resistencia a la compresión $\left(\mathrm{kp} / \mathrm{cm}^{2}\right)$

CUADRO

\begin{tabular}{|c|c|c|c|c|c|c|c|c|c|}
\hline \multirow[t]{2}{*}{$\begin{array}{c}\text { Amasada } \\
n \cong\end{array}$} & \multirow[t]{2}{*}{$\begin{array}{c}\text { Probeta } \\
n ?\end{array}$} & \multirow{2}{*}{$\begin{array}{l}\text { Moldeadas } \\
\text { cilindros } \\
\phi=15 \mathrm{~cm} \\
h=30 \mathrm{~cm}\end{array}$} & \multicolumn{2}{|c|}{$\begin{array}{l}\text { Cortadas } \\
\phi=15 \mathrm{~cm} \\
h=30 \mathrm{~cm}\end{array}$} & \multicolumn{2}{|c|}{$\begin{array}{l}\text { Cortadas } \\
\phi=10 \mathrm{~cm} \\
h=20 \mathrm{~cm}\end{array}$} & \multicolumn{2}{|c|}{$\begin{array}{l}\text { Cortadas } \\
\phi=5 \mathrm{~cm} \\
h=10 \mathrm{~cm}\end{array}$} & \multirow{2}{*}{$\begin{array}{l}\text { Moldeadas } \\
\text { prismas } \\
\square ~ 20 \times 20 \mathrm{~cm} \text {. } \\
h=50 \mathrm{~cm} .\end{array}$} \\
\hline & & & Hor. & Vert. & Hor. & Vert. & Hor. & vert. & \\
\hline & & I & I I & III & IV & V & VI & VII & VIII \\
\hline 1 & $\begin{array}{l}1 \\
2 \\
3 \\
4 \\
5 \\
6\end{array}$ & $\begin{array}{l}129,0 \\
139,5 \\
131,0 \\
145,0 \\
139,0 \\
144,0\end{array}$ & $\begin{array}{l}141,5 \\
146,5 \\
147,0 \\
150,0 \\
139,5 \\
153,0\end{array}$ & $\begin{array}{l}150,0 \\
149,5 \\
158,0 \\
153,0 \\
156,5 \\
150,0\end{array}$ & $\begin{array}{l}140,5 \\
152,5 \\
146,5 \\
150,0 \\
147,0 \\
141,5\end{array}$ & $\begin{array}{l}141,5 \\
155,0 \\
163,0 \\
135,5 \\
161,5 \\
158,0\end{array}$ & $\begin{array}{l}152,5 \\
160,5 \\
114,0 \\
159,0 \\
144,5 \\
178,5\end{array}$ & $\begin{array}{l}114,5 \\
148,0 \\
134,5 \\
143,5 \\
160,5 \\
145,5\end{array}$ & $\begin{array}{l}139,7 \\
134,0\end{array}$ \\
\hline 2 & $\begin{array}{r}7 \\
8 \\
9 \\
10 \\
11 \\
12 \\
\end{array}$ & $\begin{array}{l}104,5 \\
114,5 \\
117,5 \\
112,0 \\
114,5 \\
111,0\end{array}$ & $\begin{array}{l}143,5 \\
128,0 \\
138,5 \\
141,0 \\
131,5 \\
146,5\end{array}$ & $\begin{array}{l}136,5 \\
126,5 \\
135,5 \\
137,5 \\
134,5 \\
149,0\end{array}$ & $\begin{array}{l}150,5 \\
145,5 \\
148,5 \\
139,0 \\
125,0 \\
151,0\end{array}$ & $\begin{array}{l}141,5 \\
131,5 \\
155,0 \\
139,0 \\
143,0 \\
152,5\end{array}$ & $\begin{array}{l}154,5 \\
124,5 \\
125,0 \\
152,0 \\
138,5 \\
152,0\end{array}$ & $\begin{array}{l}106,5 \\
125,0 \\
158,0 \\
134,5 \\
163,0 \\
136,5\end{array}$ & $\begin{array}{l}142,2 \\
140,5\end{array}$ \\
\hline 3 & $\begin{array}{l}13 \\
14 \\
15 \\
16 \\
17 \\
18\end{array}$ & $\begin{array}{l}113,0 \\
112,5 \\
119,0 \\
118,5 \\
115,5 \\
125,0\end{array}$ & $\begin{array}{l}134,0 \\
134,0 \\
142,5 \\
140,0 \\
142,0 \\
136,5\end{array}$ & $\begin{array}{l}132,0 \\
141,5 \\
144,5 \\
141,5 \\
160,5 \\
161,5\end{array}$ & $\begin{array}{l}155,0 \\
141,0 \\
142,5 \\
136,5 \\
108,5 \\
130,0\end{array}$ & $\begin{array}{l}134,5 \\
143,5 \\
149,5 \\
118,0 \\
132,0 \\
143,5\end{array}$ & $\begin{array}{r}126,0 \\
143,0 \\
127,0 \\
120,0 \\
96,0 \\
134,0\end{array}$ & $\begin{array}{r}95,0 \\
104,0 \\
140,5 \\
90,5 \\
116,0 \\
115,5\end{array}$ & $\begin{array}{l}158,0 \\
131,7\end{array}$ \\
\hline 4 & $\begin{array}{l}19 \\
20 \\
21 \\
22 \\
23 \\
24 \\
\end{array}$ & $\begin{array}{l}134,9 \\
124,7 \\
121,2 \\
130,2 \\
116,8 \\
116,1 \\
\end{array}$ & $\begin{array}{l}148,0 \\
143,3 \\
153,5 \\
152,2 \\
138,8 \\
145,2\end{array}$ & $\begin{array}{l}153,5 \\
138,8 \\
148,2 \\
151,2 \\
151,6 \\
145,9\end{array}$ & $\begin{array}{l}144,0 \\
160,7 \\
143,0 \\
146,2 \\
146,2 \\
163,5\end{array}$ & $\begin{array}{l}165,0 \\
160,0 \\
168,9 \\
162,3 \\
142,8 \\
175,5\end{array}$ & $\begin{array}{l}135,2 \\
122,0 \\
147,5 \\
128,2 \\
129,0 \\
128,7\end{array}$ & $\begin{array}{l}135,2 \\
135,0 \\
141,7 \\
128,8 \\
141,9 \\
129,1\end{array}$ & $\begin{array}{l}168,3 \\
164,0\end{array}$ \\
\hline 5 & $\begin{array}{l}25 \\
26 \\
27 \\
28 \\
29 \\
30 \\
\end{array}$ & $\begin{array}{l}143,5 \\
137,0 \\
135,5 \\
140,5 \\
142,5 \\
129,5 \\
\end{array}$ & $\begin{array}{l}105,0 \\
132,0 \\
141,0 \\
146,5 \\
144,0 \\
132,0\end{array}$ & $\begin{array}{l}157,0 \\
140,5 \\
164,5 \\
174,0 \\
164,0 \\
154,5 \\
\end{array}$ & $\begin{array}{l}135,5 \\
145,0 \\
138,0 \\
124,5 \\
133,5 \\
118,5\end{array}$ & $\begin{array}{l}150,5 \\
145,5 \\
161,0 \\
142,0 \\
167,5 \\
143,0 \\
\end{array}$ & $\begin{array}{l}176,0 \\
157,0 \\
121,5 \\
144,0 \\
131,5 \\
118,5\end{array}$ & $\begin{array}{l}146,0 \\
154,0 \\
135,0 \\
160,5 \\
116,5 \\
112,5 \\
\end{array}$ & $\begin{array}{l}158,7 \\
165,7\end{array}$ \\
\hline & $\begin{array}{l}\text { Valor } \\
\text { medio }\end{array}$ & 125,9 & 140,6 & 148,7 & 141,6 & 149,4 & 138.0 & 132,3 & 150,3 \\
\hline & $\begin{array}{c}\text { Coef. } \\
\text { variación \% }\end{array}$ & 10,2 & 6,6 & 7,3 & 8,4 & 8,8 & 14,7 & 32,0 & 9,3 \\
\hline & Res./I & 1. & 1,12 & 1,18 & 1,12 & 1,19 & 1,10 & 1,05 & 1,19 \\
\hline
\end{tabular}


efecto del corte y al tamaño de probeta que al ser reducido se acerca al tamaño de la "estructura" del hormigón.

2. En cuanto a descenso del valor medio de la resistencia por el efecto del corte pueden sacarse algunas conclusiones a partir del cuadro 4.

En él están calculados los factores por los que hay que multiplicar las resistencias obtenidas sobre un tamaño de testigo cualquiera, para igualar la de los testigos de $15 \mathrm{~cm}$ de diámetro, que por su tamaño se han supuesto de deterioro despreciable.

MASA DE $200 \mathrm{kp} / \mathrm{cm}^{2}$

Resistencia a la compresión $\left(\mathrm{kp} / \mathrm{cm}^{2}\right)$

CUADRO

\begin{tabular}{|c|c|c|c|c|c|c|c|c|c|}
\hline \multirow[t]{2}{*}{$\begin{array}{c}\text { Amasada } \\
n \cong\end{array}$} & \multirow[t]{2}{*}{$\begin{array}{l}\text { Probeta } \\
n \cong\end{array}$} & \multirow{2}{*}{$\begin{array}{l}\text { Maldeadas } \\
\text { cilindros } \\
\phi=15 \mathrm{~cm} \\
h=30 \mathrm{~cm}\end{array}$} & \multicolumn{2}{|c|}{$\begin{array}{l}\text { Cortadas } \\
\phi=15 \mathrm{~cm} \\
h=30 \mathrm{~cm}\end{array}$} & \multicolumn{2}{|c|}{$\begin{array}{l}\text { Cortadas } \\
\phi=10 \mathrm{~cm} \\
h=20 \mathrm{~cm}\end{array}$} & \multicolumn{2}{|c|}{$\begin{array}{l}\text { Cortadas } \\
\phi=5 \mathrm{~cm} \\
h=10 \mathrm{~cm}\end{array}$} & \multirow{2}{*}{$\begin{array}{l}\text { Moldeadas } \\
\text { prismas } \\
\square 20 \times 20 \mathrm{~cm} \text {. } \\
h=50 \mathrm{~cm} .\end{array}$} \\
\hline & & & Hor. & vert. & Hor. & Vert. & Hor. & vert. & \\
\hline & & I & I I & I I I & IV & v & VI & VII & VIII \\
\hline 1 & $\begin{array}{l}1 \\
2 \\
3 \\
4 \\
5 \\
6\end{array}$ & $\begin{array}{l}215,0 \\
179,0 \\
203,0 \\
190,0 \\
204,0 \\
174,0\end{array}$ & $\begin{array}{l}213,0 \\
180,5 \\
196,5 \\
191,5 \\
180,5 \\
192,0 \\
\end{array}$ & $\begin{array}{l}242,0 \\
245,0 \\
232,0 \\
245,5 \\
221,0 \\
209,0 \\
\end{array}$ & $\begin{array}{l}186,5 \\
171,5 \\
166,0 \\
158,5 \\
161,5 \\
187,5\end{array}$ & $\begin{array}{l}243,0 \\
262,5 \\
205,0 \\
244,5 \\
238,0 \\
244,5\end{array}$ & $\begin{array}{l}183,5 \\
209,0 \\
205,5 \\
150,5 \\
186,0 \\
173,5\end{array}$ & $\begin{array}{l}241,0 \\
255,0 \\
147,5 \\
233,0 \\
272,0 \\
205,0\end{array}$ & $\begin{array}{l}201,2 \\
211,0\end{array}$ \\
\hline 2 & $\begin{array}{l}7 \\
8 \\
9 \\
10 \\
11 \\
12\end{array}$ & $\begin{array}{l}182,5 \\
185,0 \\
213,0 \\
181,5 \\
185,5 \\
188,5\end{array}$ & $\begin{array}{l}228,0 \\
237,0 \\
227,5 \\
221,5 \\
212,0 \\
219,5\end{array}$ & $\begin{array}{l}210,0 \\
209,5 \\
237,0 \\
229,0 \\
213,5 \\
209,5\end{array}$ & $\begin{array}{l}205,5 \\
205,0 \\
198,5 \\
206,0 \\
199,5 \\
216,5\end{array}$ & $\begin{array}{l}239,0 \\
207,0 \\
236,5 \\
206,5 \\
230,0 \\
234,5\end{array}$ & $\begin{array}{l}188,5 \\
270,5 \\
167,0 \\
215,5 \\
219,5 \\
200,0\end{array}$ & $\begin{array}{l}168,0 \\
147,0 \\
235,5 \\
163,0 \\
189,0 \\
208,5\end{array}$ & $\begin{array}{l}221,2 \\
201,2\end{array}$ \\
\hline 3 & $\begin{array}{l}13 \\
14 \\
15 \\
16 \\
17 \\
18 \\
\end{array}$ & $\begin{array}{l}195,5 \\
203,0 \\
201,0 \\
191,0 \\
185,5 \\
210,0\end{array}$ & $\begin{array}{l}197,5 \\
203,5 \\
199,0 \\
193,0 \\
217,0 \\
216,5\end{array}$ & $\begin{array}{l}190,5 \\
241,0 \\
247,5 \\
236,0 \\
255,0 \\
229,5\end{array}$ & $\begin{array}{l}187,0 \\
190,0 \\
188,5 \\
191,5 \\
187,0 \\
181,0\end{array}$ & $\begin{array}{l}279,0 \\
257,5 \\
199,5 \\
262,5 \\
191,0 \\
253,0\end{array}$ & $\begin{array}{l}202,5 \\
215,5 \\
145,5 \\
178,0 \\
164,0 \\
170,5\end{array}$ & $\begin{array}{l}222,0 \\
198,0 \\
233,5 \\
211,0 \\
183,0 \\
218,5\end{array}$ & $\begin{array}{l}221,5 \\
210,7\end{array}$ \\
\hline 4 & $\begin{array}{l}19 \\
20 \\
21 \\
22 \\
23 \\
24\end{array}$ & $\begin{array}{l}180,5 \\
204,5 \\
204,0 \\
212,0 \\
185,0 \\
205,0\end{array}$ & $\begin{array}{l}201,0 \\
208,0 \\
213,5 \\
222,0 \\
231,0 \\
212,5\end{array}$ & $\begin{array}{l}227,0 \\
226,5 \\
226,0 \\
212,5 \\
212,0 \\
212,5\end{array}$ & $\begin{array}{l}204,5 \\
212,0 \\
216,5 \\
189,5 \\
209,0 \\
207,0\end{array}$ & $\begin{array}{l}228,5 \\
224,0 \\
224,5 \\
242,0 \\
212,0 \\
251,0\end{array}$ & $\begin{array}{l}228,0 \\
198,0 \\
122,5 \\
220,0 \\
174,5 \\
220,5\end{array}$ & $\begin{array}{l}225,0 \\
162,0 \\
173,0 \\
154,0 \\
143,5 \\
229,0\end{array}$ & $\begin{array}{l}204,5 \\
200,0\end{array}$ \\
\hline 5 & $\begin{array}{l}25 \\
26 \\
27 \\
28 \\
29 \\
30\end{array}$ & $\begin{array}{l}189,5 \\
192,0 \\
195,5 \\
188,5 \\
184,0 \\
191,0\end{array}$ & $\begin{array}{l}211,0 \\
217,5 \\
219,5 \\
209,0 \\
210,5 \\
216,5\end{array}$ & $\begin{array}{l}229,0 \\
253,0 \\
244,0 \\
248,0 \\
239,0 \\
239,5\end{array}$ & $\begin{array}{l}214,5 \\
224,0 \\
224,5 \\
206,0 \\
229,5 \\
211,5\end{array}$ & $\begin{array}{l}232,0 \\
251,5 \\
266,0 \\
275,5 \\
268,0 \\
255,0\end{array}$ & $\begin{array}{l}173,5 \\
208,0 \\
191,0 \\
177,0 \\
196,0 \\
200,0\end{array}$ & $\begin{array}{l}229,5 \\
233,5 \\
183,5 \\
248,0 \\
209,0 \\
211,0\end{array}$ & $\begin{array}{l}252,0 \\
229,2\end{array}$ \\
\hline & $\begin{array}{l}\text { Valor } \\
\text { medio }\end{array}$ & 194,0 & 210,3 & 229,0 & 197,9 & 238,8 & 191,8 & 204,4 & 215,2 \\
\hline & $\begin{array}{l}\text { Coef. } \\
\text { variación\% }\end{array}$ & 5,8 & 3,5 & 7,0 & 9,2 & 9,6 & 15,0 & 17,2 & 7,6 \\
\hline & Res./I & 1 & 1,08 & 1.18 & 1.02 & 1,23 & 0,99 & 1,05 & 1.11 \\
\hline
\end{tabular}


FACTORES POR LOS QUE HAY QUE MULTIPLICAR LAS RESISTENCIAS OBTENIDAS SOBRE TESTIGOS CORTADOS $Y$ PROBETAS MOLDEADAS PARA IGUALAR LA DE LOS TESTIGOS CILINDRICOS CORTADOS DE $15 \times 30 \mathrm{~cm}$

\begin{tabular}{|c|c|c|c|c|c|c|}
\hline $\begin{array}{c}\text { Masa } \\
\left(\mathrm{kp} / \mathrm{cm}^{2}\right)\end{array}$ & $\begin{array}{c}\text { DIRECCION } \\
\text { DE } \\
\text { HORMIGONADO }\end{array}$ & $\begin{array}{c}\text { Testigo } \\
\text { cortado } \\
\phi=15 \mathrm{~cm} \\
\mathrm{~h}=30 \mathrm{~cm}\end{array}$ & 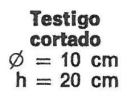 & $\begin{array}{c}\text { Testigo } \\
\text { cortado } \\
\phi=5 \mathrm{~cm} \\
\mathrm{~h}=10 \mathrm{~cm}\end{array}$ & $\begin{array}{c}\text { Cilindro } \\
\text { moldeado } \\
\varnothing=15 \mathrm{~cm} \\
\mathrm{~h}=30 \mathrm{~cm}\end{array}$ & $\begin{array}{c}\begin{array}{c}\text { Prisma } \\
\text { moldeado } \\
\square=20 \times 20 \mathrm{~cm} \\
\mathrm{~h}=50 \mathrm{~cm}\end{array}\end{array}$ \\
\hline \multirow{2}{*}{90} & Horizontal & 1,00 & 0,94 & 1,00 & 1,07 & 1,04 \\
\hline & Vertical & 1,00 & 1,01 & 1,11 & - & 1,04 \\
\hline \multirow{2}{*}{130} & Horizontal & 1,00 & 0,99 & 1,02 & 1,12 & 0,94 \\
\hline & Vertical & 1,00 & 1,00 & 1,12 & - & 0,99 \\
\hline \multirow{2}{*}{200} & Horizontal & 1,00 & 1,06 & 1,10 & 1,08 & 0,98 \\
\hline & Vertical & 1,00 & 0,96 & 1,12 & - & 1,06 \\
\hline \multicolumn{2}{|c|}{ Factores de conversión teóricos } & 1,00 & 0,97 & 0,92 & 1,00 & 1,06 \\
\hline
\end{tabular}

La diferencia para los testigos cortados de 10 y $5 \mathrm{~cm}$ de diámetro, entre los factores reales obtenidos y los teóricos (es decir los que dan las Normas y Bibliografía para paso de resistencia de un tamaño de probeta a otro), nos da una idea del deterioro en resistencia producido por el corte de los mismos. Realizando los cálculos oportunos resultan los siguientes porcentajes de deterioro:

CUADRO

\begin{tabular}{|c|c|c|c|c|c|}
\hline \multirow{3}{*}{$\begin{array}{c}\text { Masa } \\
\left(\mathrm{kp} / \mathrm{cm}^{2}\right)\end{array}$} & \multirow{3}{*}{$\begin{array}{c}\text { DIRECGION } \\
\text { DE } \\
\text { HORMIGONADO }\end{array}$} & \multicolumn{4}{|c|}{ DETERIORO EN RESISTENCIA, EN \%, RESPECTO A LA DEL TESTIGO } \\
\hline & & \multicolumn{2}{|c|}{ Testigo de $10 \mathrm{~cm}$ de $\varnothing$} & \multicolumn{2}{|c|}{ Testigo de $5 \mathrm{~cm}$ de $\varnothing$} \\
\hline & & $\begin{array}{l}\text { Según dirección } \\
\text { de hormigonado }\end{array}$ & Valor medio & $\begin{array}{l}\text { Según dirección } \\
\text { de hormigonado }\end{array}$ & Valor medio \\
\hline \multirow{2}{*}{90} & Horizontal & $-3,1$ & \multirow{2}{*}{0,5} & 8,6 & \multirow{2}{*}{14,6} \\
\hline & Vertical & 4,1 & & 20,6 & \\
\hline \multirow{2}{*}{130} & Horizontal & 2,0 & \multirow{2}{*}{2,6} & 10,9 & \multirow{2}{*}{16,3} \\
\hline & Vertical & 3,1 & & 21,7 & \\
\hline \multirow{2}{*}{200} & Horizontal & 9,3 & \multirow{2}{*}{4,2} & 19,5 & \multirow{2}{*}{20,6} \\
\hline & Vertical & $-1,0$ & & 21,7 & \\
\hline
\end{tabular}

De nuestras experiencias se deduce que los deterioros medios en el corte de testigos de $10 \mathrm{~cm}$ de diámetro son pequeños y afectan a la resistencia en valores que van de 0,5 a $4,2 \%$, correspondiéndose mayor deterioro a mejor calidad del hormigón. La relación en este caso entre diámetro de testigo y tamaño máximo del árido es de cuatro.

Sobre testigo cortado de $5 \mathrm{~cm}$ de diámetro el deterioro ya es grande y oscila entre el 14,6 y el 20,6\%, aumentando también con la calidad del hormigón. Para este tamaño de probeta la relación entre diámetro de testigo y tamaño máximo del árido es de dos.

Nos ha sorprendido, pues es contrario a la idea que teníamos en un principio y que presentaba su lógica física, el que el deterioro de resistencia sea creciente con la calidad del hormigón. 
Parecía más lógico que los hormigones de poca calidad quedaran más influenciados por los efectos mecánicos del corte que los de buena calidad. Sin embargo, eso es lo que demuestran los resultados y supone una curiosa constatación.

Se puede añadir también la total idoneidad de los testigos cortados de $10 \mathrm{~cm}$ de diámetro, que dañan menos que los de $15 \mathrm{~cm}$ de diámetro al elemento estructural de que se extraen, para el control de resistencia de estructuras terminadas.

En efecto, de los anteriores resultados se desprenden unas correcciones muy pequeñas en cuanto a deterioro por el corte, y del punto $1 .^{\circ}$ de estas conclusiones se deduce la pequeña dispersión de los resultados obtenidos. La resistencia indicada por estos testigos representa con fidelidad a la de la obra.

Con los testigos de $5 \mathrm{~cm}$ de diámetro la representatividad de la obra puede quedar bastante disminuida, tanto por la alta dispersión de sus resultados como por la importante corrección que precisan debido al fuerte deterioro del corte.

3. Las probetas cilíndricas de Normas, moldeadas a la vez que las piezas de las que se han sacado testigos con trépano, y conservadas de igual forma en el ambiente del laboratorio, han dado valores menores de resistencia que los testigos de $15 \mathrm{~cm}$ de diámetro cortados de dichas piezas.

De la última fila de los tres cuadros generales de resultados puede comprobarse que la mejora de resistencia de los testigos sacados de la pieza hormigonada horizontalmente, de la misma altura que la probeta $(30 \mathrm{~cm})$, oscila entre el 7 y el $12 \%$. La mejora de los testigos sacados de la pieza hormigonada verticalmente, de $110 \mathrm{~cm}$ de altura, es todavía mayor y comprendida entre el 8 y $18 \%$.

Esto será debido, probablemente, a una mejor compactación del hormigón de los elementos grandes tipo obra respecto a la de las probetas moldeadas de tamaño más reducido, como lo atestigua la mayor ganancia de resistencia de la pieza más alta.

Se deduce, por tanto, que a iguales condiciones de conservación y curado la resistencia de la obra será superior, dentro de los órdenes de valores antes manejados, a la de las probetas moldeadas durante su construcción. Pero para ello, insistimos, probetas y obra debieran tener el mismo tratamiento. (Exceptuamos en esta consideración la parte superior de los pilares, sobre todo de mala calidad, cuya resistencia puede llegar a ser inferior a la de las probetas moldeadas).

Lamentablemente no se cuida en las obras en general el aspecto del curado y entonces no se puede hablar de mejora de resistencia respecto a las probetas moldeadas en obra y curadas en laboratorio según Normas, habiendo autores $(2,5$ y 6) que establecen que la resistencia de estas últimas es incluso superior en este caso a la del hormigón de la obra.

4. El coeficiente medio de conversión de la resistencia de los prismas de $20 \times 20 \times 50 \mathrm{~cm}$ a cilindro de $15 \times 30 \mathrm{~cm}$ nos sale 1,11 en vez de 1,06, deducido de algunas tablas de uso corriente en ensayo de hormigón.

\section{influencia de la extracción de un testigo y relleno posterior en la resistencia a la compresión de la pieza perforada}

\subsection{PLANTEAMIENTO DE LA INVESTIGACION}

El objeto de la investigación, en esta segunda parte del trabajo, ha sido verificar la pérdida de resistencia a la compresión de un pilar al que, después de haber sido perforado para extracción de un testigo cilíndrico de $10 \mathrm{~cm}$. de diámetro, se rellena el agujero de distintas maneras quedando resistiendo de esa forma. 
Como en el caso anterior se han repetido todos los ensayos en hormigones de resistencias nominales a 90 días de 90,130 y $200 \mathrm{kp} / \mathrm{cm}^{2}$.

La influencia del material de relleno se ha puesto de manifiesto utilizando en un caso hormigón sin adición alguna, en otro hormigón con un ligero expansivo y, finalmente, hormigón con el expansivo pero dando a las paredes del agujero resina epoxi para procurar el perfecto pegado del relleno con las paredes de la pieza perforada.

El conjunto de resultados que se han obtenido de los diferentes ensayos, para cada calidad de hormigón, comprende:

Resistencias de prismas de $25 \times 25 \times 50 \mathrm{~cm}$, sin perforar.

Resistencias de prismas de $25 \times 25 \times 50 \mathrm{~cm}$, con perforaciones de $10 \mathrm{~cm}$ de diámetro rellenas por los tres métodos antes descritos.

Resistencias de los testigos cilíndricos extraídos de los prismas perforados, de $10 \mathrm{~cm}$ de diámetro y $25 \mathrm{~cm}$ de altura, aproximadamente.

El proceso de preparación de las probetas ha sido el siguiente:

De cada uno de los niveles de calidad del hormigón se han realizado 18 masas, moldeando con cada una dos prismas gemelos de $25 \times 25 \times 50 \mathrm{~cm}$.

A los $60^{\circ}$ días aproximadamente se perforaba en la mitad de su altura y centrado con las caras laterales, por corte con corona de polvo de diamante, un agujero de $10 \mathrm{~cm}$ de diámetro, con extracción del testigo correspondiente. Dos días después se rellenaba por el procedimiento que correspondiese el agujero. Se efectuaron seis ensayos por cada método de relleno (foto 3).

A los 90 días, y por lo tanto 28 desde el momento del relleno, se rompían, a compresión, los prismas gemelos, con y sin perforación, y el testigo extraído del perforado (fotos 4,5 y 6 ).

Por tanto, después de las roturas a la edad de 90 días hemos dispuesto, para cada nivel de resistencia, de seis parejas de valores de comparación de cargas de rotura a compresión entre prismas sin perforar y perforados y luego rellenos por cada uno de los diferentes procedimientos.

\subsection{DATOS TECNICOS CORRESPONDIENTES A LOS ENSAYOS}

\subsubsection{Aridos}

Idéntico al apartado 1.2.1.

\subsubsection{Características de las masas}

Las masas de calidad resistente nominal a 90 días, de 90,130 y $200 \mathrm{kp} / \mathrm{cm}^{2}$, idénticas a las indicadas en el apartado 1.2.2. 

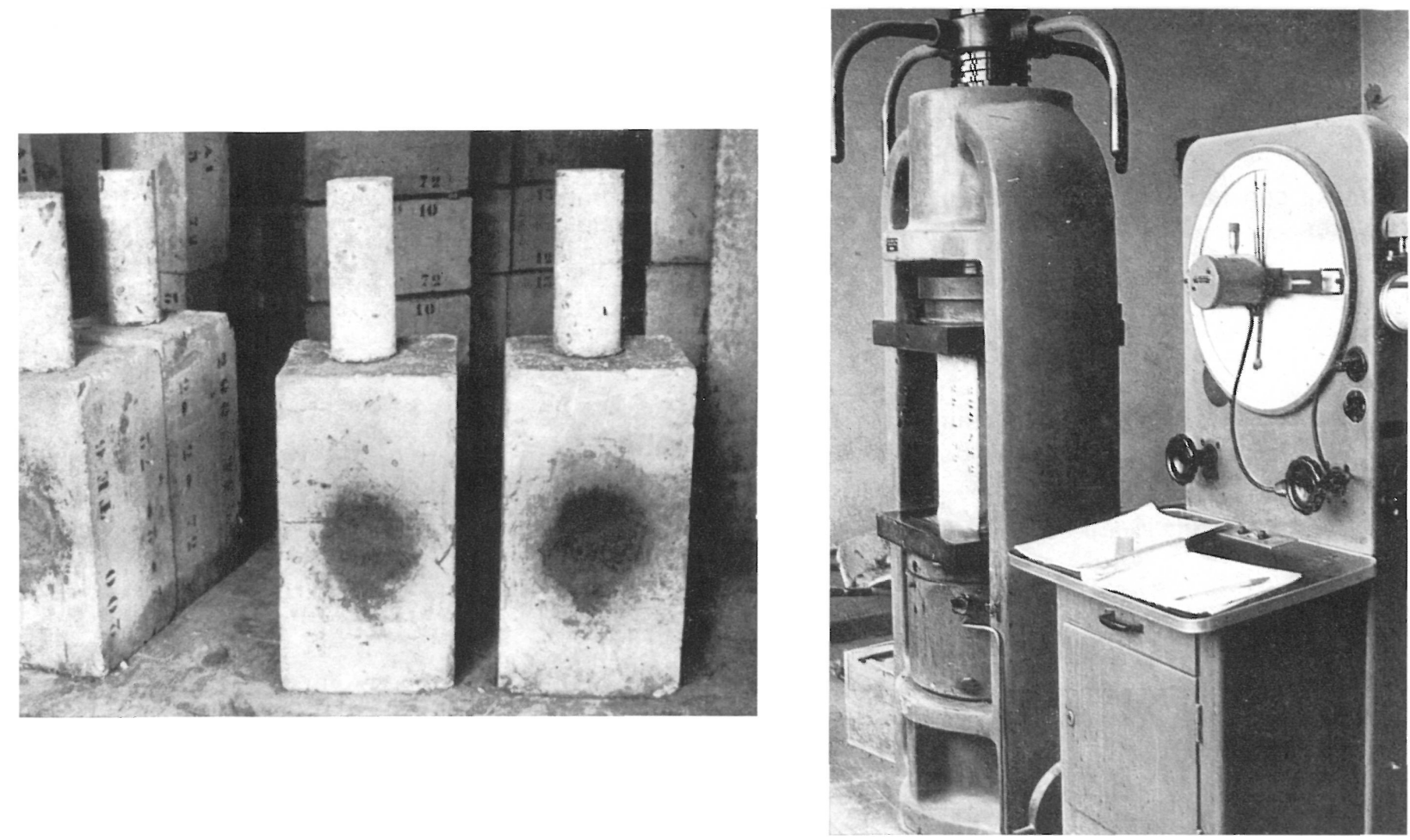

Foto 3. Prismas perforados, una vez efectuado el relleno.

Foto 4. Ensayo de prismas en una prensa Amsler.

Foto 5. Rotura de prisma con relleno.

Foto 6. Rotura mostrando la no adherencia de prisma y relleno.
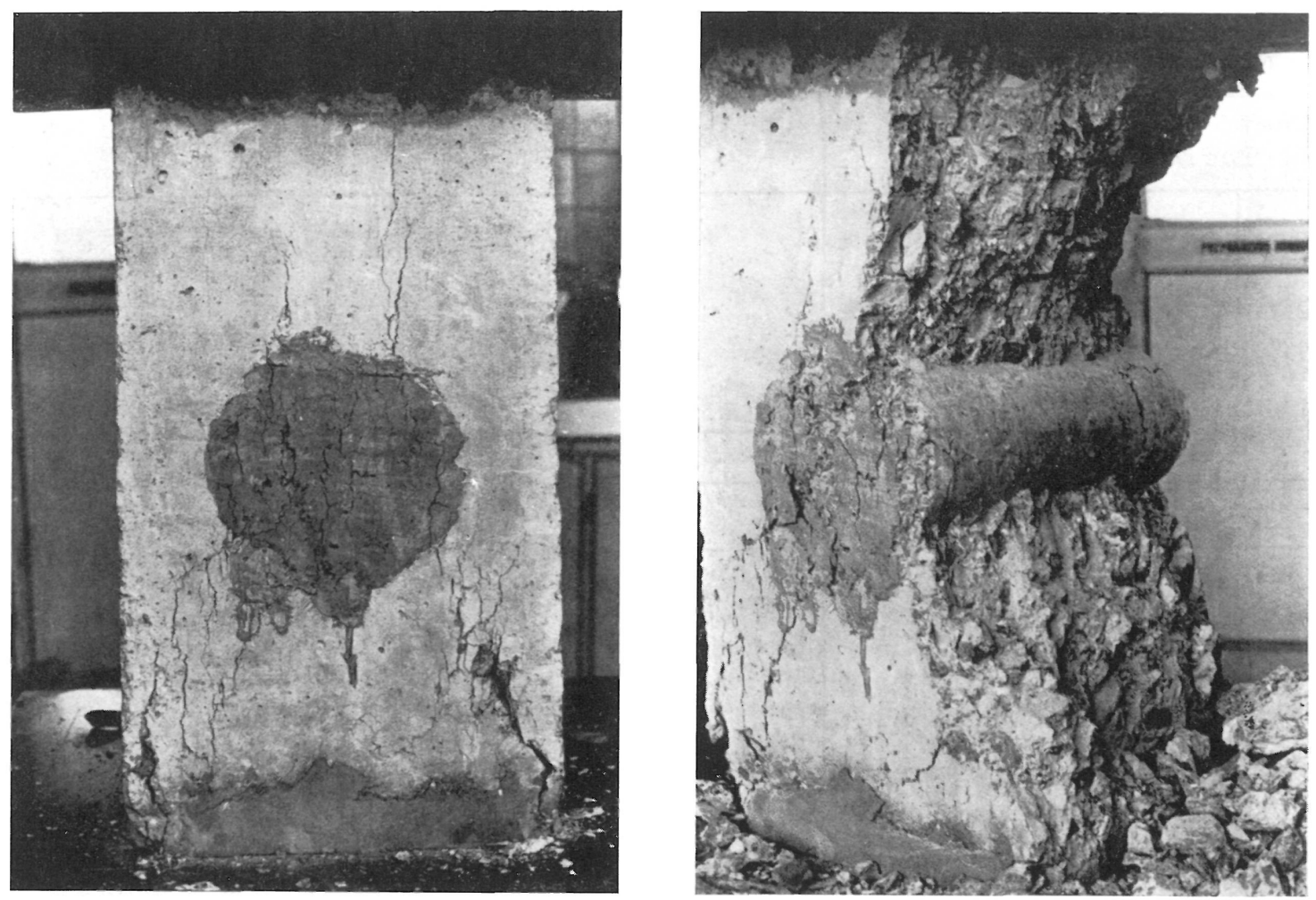
La masa con la que se rellenaba los 62 días el prisma perforado era de las características siguientes:

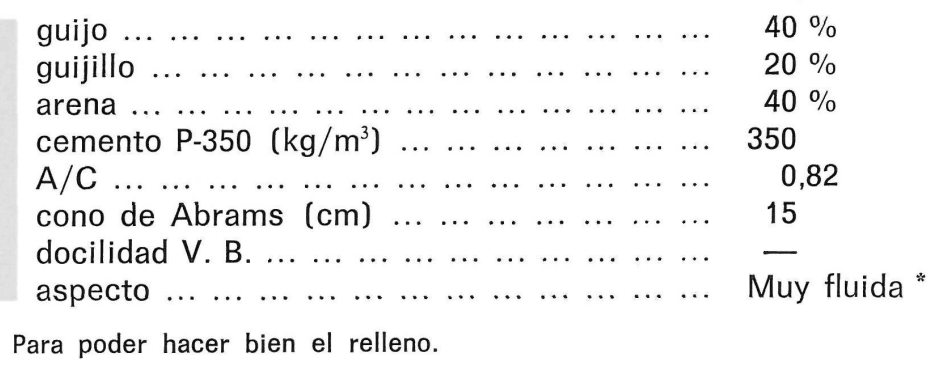

MASA DE $90 \mathrm{kp} / \mathrm{cm}^{2}$

CUADRO Resistencia a la compresión $\left(\mathrm{kp} / \mathrm{cm}^{2}\right)$

\begin{tabular}{|c|c|c|c|c|c|c|}
\hline \multirow[b]{2}{*}{ Grupo } & \multirow[b]{2}{*}{$\left|\begin{array}{c}\text { Probeta } \\
n \cong\end{array}\right|$} & \multirow[b]{2}{*}{$\begin{array}{l}\text { Prismas de } \\
25 \times 25 \times 50 \mathrm{~cm} \\
\text { sin perforar }\end{array}$} & \multicolumn{3}{|c|}{$\begin{array}{c}\text { Prismas de } 25 \times 25 \times 50 \mathrm{~cm} \text {, con } \\
\text { perforación transversal de } \\
10 \mathrm{~cm}, \varnothing \text { relleno con: }\end{array}$} & \multirow{2}{*}{$\begin{array}{l}\text { Testigos } \\
\text { extraidos de las } \\
\text { perforaciones: } \\
\emptyset=10 \mathrm{~cm} . \\
h=25 \mathrm{~cm} .\end{array}$} \\
\hline & & & $\begin{array}{c}\text { Hormigón } \\
\text { normal }\end{array}$ & $\begin{array}{l}\text { Hormigón } \\
\text { con } \\
\text { expansivo }\end{array}$ & $\begin{array}{l}\text { Hormigón con } \\
\text { expansivoy } \\
\text { epoxy }\end{array}$ & \\
\hline 1 & $\begin{array}{l}1 \\
2 \\
3 \\
4 \\
5 \\
6\end{array}$ & $\begin{array}{r}-- \\
103,4 \\
92,6 \\
100,2 \\
106,1 \\
96,8\end{array}$ & $\begin{array}{r}-- \\
89,7 \\
88,7 \\
103,6 \\
96,2 \\
93,2\end{array}$ & & & $\begin{array}{l}129,5 \\
115,0 \\
106,5 \\
116,5 \\
113,5 \\
119,3\end{array}$ \\
\hline \multicolumn{2}{|c|}{ Medias } & 99,8 & 94,3 & & & 116,7 \\
\hline \multicolumn{2}{|c|}{$\begin{array}{l}\text { Coef. de } \\
\text { variación( } \%)\end{array}$} & 5,3 & 6,4 & & & 6,5 \\
\hline 2 & $\begin{array}{r}7 \\
8 \\
9 \\
10 \\
11 \\
12\end{array}$ & $\begin{array}{r}110,7 \\
98,5 \\
102,7 \\
104,4 \\
94,4 \\
91,4\end{array}$ & & $\begin{array}{r}118,5 \\
88,4 \\
90,8 \\
93,6 \\
80,8 \\
88,8\end{array}$ & & $\begin{array}{l}138,2 \\
113,5 \\
154,2 \\
139,8 \\
110,7 \\
107,8\end{array}$ \\
\hline \multicolumn{2}{|c|}{ Medias } & 100,3 & & 93,5 & & 127,4 \\
\hline \multicolumn{2}{|c|}{$\begin{array}{l}\text { Coef. de } \\
\text { variación(\%) }\end{array}$} & 7,0 & & 12,5 & & 15,0 \\
\hline 3 & $\begin{array}{l}13 \\
14 \\
15 \\
16 \\
17 \\
18 \\
\end{array}$ & $\begin{array}{r}129,6 \\
89,4 \\
78,6 \\
91,1 \\
87,1 \\
83,1 \\
\end{array}$ & & & $\begin{array}{r}115,9 \\
71,0 \\
85,4 \\
83,2 \\
86,9 \\
87,5 \\
\end{array}$ & $\begin{array}{r}149,5 \\
104,0 \\
97,5 \\
110,5 \\
96,2 \\
94,7 \\
\end{array}$ \\
\hline \multicolumn{2}{|c|}{ Medias } & 93,2 & & & 88,3 & 108,7 \\
\hline \multicolumn{2}{|c|}{$\begin{array}{l}\text { Coef. de } \\
\text { variación(\%) }\end{array}$} & 19,8 & & & 16,8 & 19,2 \\
\hline
\end{tabular}


En un segundo método de relleno del agujero se añadía a la masa anterior un producto ligeramente expansivo para contrarrestar la retracción, en la proporción del $2 \%$ respecto al cemento.

En el tercer método de relleno, la masa con el expansivo se colaba en el agujero cuyas paredes habían sido previamente preparadas con resina epoxi del tipo de dos componentes y mezcla en frío.

Para conocer las características de la masa de relleno, con y sin expansivo, se hicieron masas de acuerdo con las proporciones anteriores, de las que se moldearon probetas cilíndricas que en el ensayo a compresión dieron los resultados del cuadro 9.

MASA DE $130 \mathrm{kp} / \mathrm{cm}^{2}$

Resistencia a la compresión $\left(\mathrm{kp} / \mathrm{cm}^{2}\right)$

CUADRO

\begin{tabular}{|c|c|c|c|c|c|c|}
\hline \multirow[b]{2}{*}{ Grupo } & \multirow[b]{2}{*}{$\begin{array}{c}\text { Probeta } \\
n \cong\end{array}$} & \multirow[b]{2}{*}{$\begin{array}{l}\text { Prismas de } \\
25 \times 25 \times 50 \mathrm{~cm} \\
\text { sin perforar }\end{array}$} & \multicolumn{3}{|c|}{$\begin{array}{l}\text { Prismas de } 25 \times 25 \times 50 \mathrm{~cm} \text {, con } \\
\text { perforación transversal de } \\
10 \mathrm{~cm}, \varnothing \text { relleno con: }\end{array}$} & \multirow{2}{*}{$\begin{array}{l}\text { Testigos } \\
\text { extraidos de las } \\
\text { perforaciones: } \\
\varnothing=10 \mathrm{~cm} . \\
h=25 \mathrm{~cm} .\end{array}$} \\
\hline & & & $\begin{array}{c}\text { Hormigón } \\
\text { normal }\end{array}$ & $\begin{array}{l}\text { Hormigón } \\
\text { con } \\
\text { expansivo }\end{array}$ & $\begin{array}{l}\text { Hormigón con } \\
\text { expansivo y } \\
\text { epoxy }\end{array}$ & \\
\hline 1 & $\begin{array}{l}1 \\
2 \\
3 \\
4 \\
5 \\
6\end{array}$ & $\begin{array}{l}139,2 \\
136,9 \\
139,8 \\
153,2 \\
154,3 \\
166,0\end{array}$ & $\begin{array}{l}117,0 \\
130,8 \\
126,4 \\
136,0 \\
140,5 \\
166,8\end{array}$ & & & $\begin{array}{l}161,3 \\
159,6 \\
150,1 \\
165,7 \\
195,8 \\
177,2\end{array}$ \\
\hline \multicolumn{2}{|c|}{ Medias } & 148,2 & 136,2 & & & 168,4 \\
\hline \multicolumn{2}{|c|}{$\begin{array}{l}\text { Coef. de } \\
\text { variación(\%) }\end{array}$} & 7,7 & 12,5 & & & 9,6 \\
\hline 2 & $\begin{array}{r}7 \\
8 \\
9 \\
10 \\
11 \\
12\end{array}$ & $\begin{array}{l}167,7 \\
166,7 \\
146,4 \\
172,4 \\
152,1 \\
163,6\end{array}$ & & $\begin{array}{l}136,0 \\
142,9 \\
128,2 \\
146,8 \\
145,6 \\
150,3\end{array}$ & & $\begin{array}{l}177,6 \\
180,4 \\
155,6 \\
180,1 \\
152,7 \\
173,6\end{array}$ \\
\hline \multicolumn{2}{|c|}{ Medias } & 161,5 & & 141,6 & & 170,0 \\
\hline \multicolumn{2}{|c|}{$\begin{array}{l}\text { Coef. de } \\
\text { variación(\%) }\end{array}$} & 6,2 & & 5,8 & & 7,4 \\
\hline 3 & $\begin{array}{l}13 \\
14 \\
15 \\
16 \\
17 \\
18 \\
\end{array}$ & $\begin{array}{l}129,9 \\
129,7 \\
133,6 \\
146,2 \\
133,5 \\
128,6 \\
\end{array}$ & & & $\begin{array}{r}100,2 \\
110,5 \\
103,6 \\
95,2 \\
126,9 \\
109,5 \\
\end{array}$ & $\begin{array}{l}146,5 \\
143,5 \\
152,0 \\
151,5 \\
153,0 \\
141,5 \\
\end{array}$ \\
\hline \multicolumn{2}{|c|}{ Medias } & 133,6 & & & 107.6 & 148,0 \\
\hline \multicolumn{2}{|c|}{$\begin{array}{l}\text { Coef. de } \\
\text { variación }(\%)\end{array}$} & 4,9 & & & 10,2 & 3,3 \\
\hline
\end{tabular}


MASA DE $200 \mathrm{kp} / \mathrm{cm}^{2}$ Resistencia a la compresión $\left(\mathrm{kp} / \mathrm{cm}^{2}\right)$

\begin{tabular}{|c|c|c|c|c|c|c|}
\hline \multirow[b]{2}{*}{ Grupo } & \multirow[b]{2}{*}{$\mid \begin{array}{c}\text { Probeta } \\
n e\end{array}$} & \multirow[b]{2}{*}{$\begin{array}{l}\text { Prismas de } \\
25 \times 25 \times 50 \mathrm{~cm} \\
\text { sin perfora }\end{array}$} & \multicolumn{3}{|c|}{$\begin{array}{c}\text { Prismas de } 25 \times 25 \times 50 \mathrm{~cm} \text {, con } \\
\text { perforación transversal de } \\
10 \mathrm{~cm}, \varnothing \text { relleno con: }\end{array}$} & \multirow{2}{*}{$\begin{array}{l}\text { Testigos } \\
\text { extraidos de las } \\
\text { perforaciones: } \\
\phi=10 \mathrm{~cm} . \\
h=25 \mathrm{~cm} .\end{array}$} \\
\hline & & & $\begin{array}{c}\text { Hormigón } \\
\text { normal }\end{array}$ & $\begin{array}{l}\text { Hormigón } \\
\text { con } \\
\text { expansivo }\end{array}$ & $\begin{array}{l}\text { Hormigón con } \\
\text { expansivo } \\
\text { epoxy }\end{array}$ & \\
\hline 1 & $\begin{array}{l}1 \\
2 \\
3 \\
4 \\
5 \\
6\end{array}$ & $\begin{array}{l}222,9 \\
238,6 \\
217,8 \\
241,3 \\
219,8 \\
209,2\end{array}$ & $\begin{array}{l}140,8 \\
165,4 \\
184,9 \\
193,2 \\
155,5 \\
156,8\end{array}$ & & & $\begin{array}{l}239,3 \\
245,6 \\
241,3 \\
227,6 \\
261,0 \\
223,0\end{array}$ \\
\hline \multicolumn{2}{|c|}{ Medias } & 224,9 & 166,1 & & & 239,6 \\
\hline \multicolumn{2}{|c|}{$\begin{array}{l}\text { Coef. de } \\
\text { variación }(\%)\end{array}$} & 5,6 & 11,8 & & & 5,6 \\
\hline 2 & $\begin{array}{r}7 \\
8 \\
9 \\
10 \\
11 \\
12 \\
\end{array}$ & $\begin{array}{l}188,9 \\
223,3 \\
215,9 \\
218,7 \\
225,9 \\
231,8 \\
\end{array}$ & & $\begin{array}{l}183,8 \\
216,8 \\
169,5 \\
173,9 \\
199,3 \\
191,5 \\
\end{array}$ & & $\begin{array}{l}178,3 \\
206,1 \\
237,7 \\
201,1 \\
265,9 \\
265,5 \\
\end{array}$ \\
\hline \multicolumn{2}{|c|}{ Medias } & 217,4 & & 189,1 & & 225,8 \\
\hline \multicolumn{2}{|c|}{$\begin{array}{l}\text { Coef. de } \\
\text { variación(\%) }\end{array}$} & 6,9 & & 9,2 & & 16,0 \\
\hline & $\begin{array}{l}13 \\
14 \\
15 \\
16 \\
17 \\
18 \\
\end{array}$ & $\begin{array}{l}221,4 \\
198,0 \\
211,0 \\
195,0 \\
207,4 \\
212,3 \\
\end{array}$ & & & $\begin{array}{l}170,1 \\
184,2 \\
153,9 \\
153,1 \\
161,8 \\
145,4 \\
\end{array}$ & $\begin{array}{l}158,5 \\
207,0 \\
199,0 \\
195,0 \\
216,0 \\
212,0 \\
\end{array}$ \\
\hline \multicolumn{2}{|c|}{ Medias } & 207,5 & & & 161,4 & 197.9 \\
\hline \multicolumn{2}{|c|}{$\begin{array}{l}\text { Coef. de } \\
\text { variación }(\%)\end{array}$} & 4,7 & & & 8,7 & 10,6 \\
\hline
\end{tabular}

CUADRO

\begin{tabular}{|c|c|c|c|c|}
\hline \multirow{2}{*}{ MASA DE RELLENO } & \multicolumn{4}{|c|}{ RESISTENCIA A LA COMPRESION $\left(\mathrm{kp} / \mathrm{cm}^{2}\right)$} \\
\hline & \multicolumn{2}{|c|}{ Edad: 28 días } & \multicolumn{2}{|c|}{ Edad: 90 días } \\
\hline Sin expansivo & $\begin{array}{l}158,9-170,0-143,3 \\
176,2-153,1-149,8\end{array}$ & Media: 158,6 & $\begin{array}{l}217,7-245,0-239,7 \\
222,3-209,8-222,0\end{array}$ & Media: 226,1 \\
\hline Con expansivo & $\begin{array}{l}150,8-141,8-154,2 \\
141,8-150,8-156,0\end{array}$ & Media: 149,2 & $\begin{array}{l}192,5-219,9-200,2 \\
211,0-187,3-204,0\end{array}$ & Media: 202,5 \\
\hline
\end{tabular}


Como dato práctico se saca la conclusión de que el expansivo utilizado afecta en pequeña proporción a la resistencia del hormigón de relleno, máxime a la edad de 28 días, que es la que tiene cuando se ensayan los prismas "reparados". La pérdida de resistencia a dicha edad es de alrededor de un $6 \%$.

La resistencia del relleno, con o sin expansivo, se cifra en alrededor de $150 \mathrm{kp} / \mathrm{cm}^{2}$, que, si bien es apropiada para los hormigones de calidad 90 y $130 \mathrm{~kg} / \mathrm{cm}^{2}$, queda corta para el de calidad $200 \mathrm{kp} / \mathrm{cm}^{2}$. Esto ha supuesto una limitación a la hora de sacar las conclusiones, pero procurará subsanarse en futuros ensayos.

\subsubsection{Confección y conservación de probetas y testigos}

Es aplicable aquí lo dicho en el apartado 1.2 .3 con las siguientes particularidades ya indicadas, que repetimos para mayor claridad:

edad a la realización del agujero $\ldots \ldots \ldots \ldots \ldots \ldots$ días

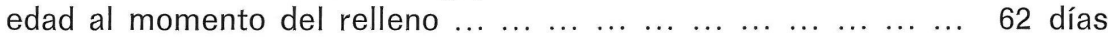

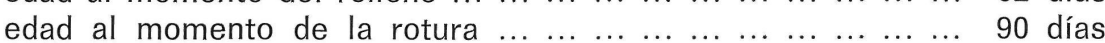

edad del relleno en el momento de la rotura de los prismas ... 28 días

\subsubsection{Resultados de los ensayos de compresión}

Aparecen recogidos en los cuadros 6,7 y 8 .

\subsection{CONCLUSIONES}

Los grados de eficacia obtenidos en las pruebas por las diferentes formas de relleno, entendiéndose por grado de eficacia el \% de resistencia alcanzado por la pieza rellena en relación con la no perforada, se encuentran resumidos en el cuadro siguiente:

GRADO DE EFICACIA DEL RELLENO (\%)

CUADRO

\begin{tabular}{|c|c|c|c|c|c|c|c|c|c|}
\hline \multirow[b]{2}{*}{$\begin{array}{ll} & \text { TIPO } \\
\text { DE } & \text { RELLENO }\end{array}$} & \multicolumn{3}{|c|}{ Masa de $90 \mathrm{kp} / \mathrm{cm}^{2}$} & \multicolumn{3}{|c|}{ Masa de $130 \mathrm{kp} / \mathrm{cm}^{2}$} & \multicolumn{3}{|c|}{ Masa de $200 \mathrm{kp} / \mathrm{cm}^{2}$} \\
\hline & Medio & Mínimo & Recorrido & Medio & Mínimo & Recorrido & Medio & Mínimo & Recorrido \\
\hline Hormigón normal & 95 & 87 & 16 & 92 & 84 & 16 & 74 & 63 & 22 \\
\hline $\begin{array}{l}\text { Hormigón } \\
\text { con expansivo }\end{array}$ & 93 & 86 & 21 & 88 & 81 & 15 & 87 & 78 & 19 \\
\hline $\begin{array}{l}\text { Hormigón } \\
\text { con expansivo y epoxi }\end{array}$ & 96 & 79 & 30 & 81 & 65 & 30 & 78 & 68 & 25 \\
\hline
\end{tabular}

De la observación del cuadro anterior se deduce lo siguiente:

1. No se ha obtenido una mejora de eficacia, considerando los resultados en su conjunto, por la utilización de expansivo en la masa de relleno y de resina epoxi en las paredes del agujero. Sin embargo, opinamos que desde el punto de vista físico debía haberse obtenido mejora, y por ello se están desarrollando nuevas series de ensayos.

Ello nos lleva a constatar el valor de las recomendaciones de la Instrucción Española de Hormigón en cuanto a las pruebas a que se deben someter los aditivos antes de su utilización. No parece ser suficiente el adoptar los productos de una marca nombrada. En este caso han fallado las previsiones de una mejora en el comportamiento del relleno. 
Foto 7. Falta de adherencia y llenado de relleno con expansivo.

Foto 8. Falta de adherencia y llenado de relleno con expansivo y epoxi.

Foto 9. Concentración de tensiones en la zona del relleno.
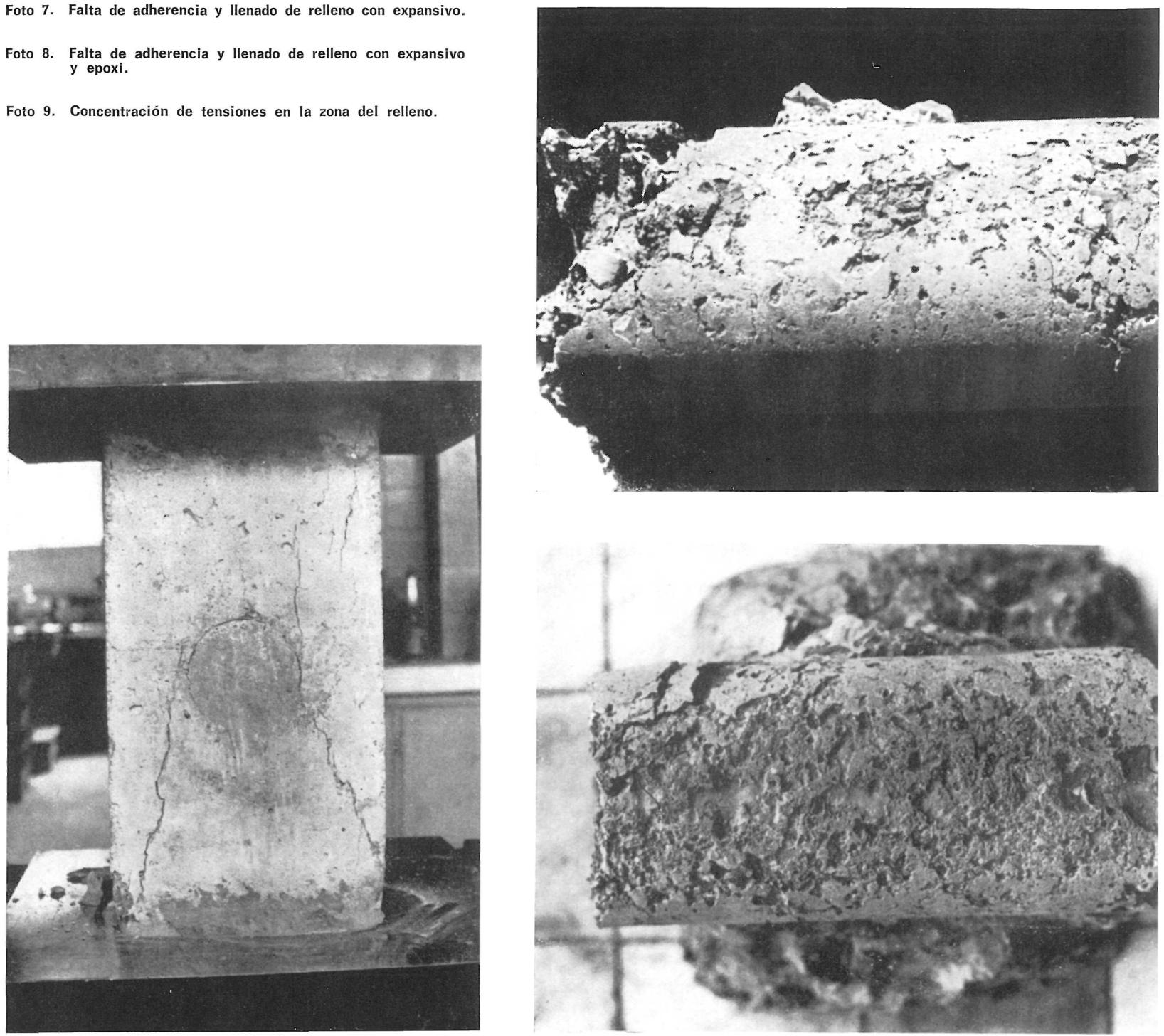

Algunas comprobaciones hechas posteriormente a los ensayos, en vista de sus resultados, han demostrado que la expansión del "expansivo" era imperceptible y, al romper los prismas con el relleno pegado con resina epoxi, éste se despegaba perfectamente del resto del hormigón (fotos 7 y 8 ).

2. Conviene hacer el relleno de la perforación con hormigón de alta resistencia. En efecto, en nuestro caso la resistencia del relleno a la edad de la rotura de los prismas era de $150 \mathrm{kp} / \mathrm{cm}^{2}$, y observamos que las eficacias disminuyen a medida que la resistencia nominal de los prismas es más elevada. En el caso del hormigón de $200 \mathrm{kp} / \mathrm{cm}^{2}$ el prisma tenía incluso resistencia superior a la del relleno.

3. No es alta la dispersión de los cocientes que miden la eficacia. En el cuadro se han incluido, además de los valores medios, los mínimos y sus recorridos. 
Rellenando el agujero, causado por el trépano, con un hormigón de alta calidad parecen esperables pérdidas de resistencia menores del $15 \%$. Para calidades de relleno poco superiores a la del elemento perforado esta pérdida puede elevarse al $20 \%$.

Claro está que estas cifras son para las dimensiones transversales del testigo $(10 \mathrm{~cm})$ y pilar $(25 \times 25 \mathrm{~cm})$ utilizadas en estos ensayos, cuya combinación es muy desfavorable. Para tamaños mayores del pilar su pérdida de resistencia será menor, en relación inversa con dicho tamaño.

4. $\quad$ La poca altura de las piezas ensayadas $\mathbf{h}=2 \mathbf{a}$ y el efecto de zunchado de los platos de la prensa ha podido concentrar el régimen de tensiones en la parte rellenada y disminuir las componentes de deslizamiento de las líneas de fuerza sobre el cilindro (foto 9).

Esto puede suponer, en el caso de que así fuera, unos resultados algo optimistas. Para precisar este efecto se están actualmente preparando nuevos ensayos con prismas de $2 \mathrm{~m}$ de altura.

Desde el punto de vista del fenómeno de cansancio pensamos que el comportamiento pueda ser mejor que el obtenido en ensayos rápidos, debido a la plasticidad del hormigón, que hará trabajar al relleno y hormigón antiguo con mayor garantía de colaboración.

\section{Bibliografía}

1. A. HOFSøY: Comparison of Apparent Compression Strength of Concrete Cores, Cubes and Cylinders. RILEM Symposium on the Experimental Research of Field Testing of Concrete. Trondheim, Noruega (1964).

2. N. PETERSONS: Strength of Concrete in Finished Estructures. RILEM Symposium on the Experimental Research of Field Testing of Concrete. Trondheim, Noruega (1964).

3. F. CAMPUS, R. DUTRON y A. COUNE: Contrôle de la qualité du béton en laboratoire et sur chantier. RILEM Symposium on the Experimental Research of Field Testing of Concrete. Trondheim, Noruega (1964).

4. R. JOOSTING: Le prélèvement et l'examen de petites carottes de béton. RILEM Symposium on the Experimental Research of Field Testing of Concrete. Trondheim, Noruega (1964).

5. A. PAVLIK: Essais du béton durcissant dans les conditions de la construction. RILEM Symposium on the Experimental Research of Field Testing of Concrete. Trondheim, Noruega (1964).

6. J. M. TOBIO: Resistencia del hormigón en estructuras terminadas. INFORMES DE LA CONSTRUCCION, número 203 , agosto-septiembre 1968

\section{résumé}

Apport sur l'évalution de la qualité résistante du béton des structures par des carottes prélevées par coupe

J. L. Ramírez Ortiz, Prof. Dr. ingénieur industriel

J. M. Bárcena Díaz, ingénieur industriel

Dans ce travail de recherche expérimentale sont étudiés deux problèmes rela-
tifs au contrôle d'ouvrage en béton durcì:

1. Celui de la détérioration subie par la résistance de la carotte en béton due à la coupe qu'il a fallu faire pour son prélèvement et le degré de representativité et de confiance fourcarottes.

2. Celui de la vérification de la perte de résistance d'éléments dont une plissage ultérieur du trou qui en a résulté.

\section{summary}

Contribution on the evaluation of the strength of concrete in buildings by means of control samples extracted by cutting

Dr. J. L. Ramírez Ortiz, Professor of Industrial Engineering

J. M. Bárcena Díaz, Industrial Engineer In this study of experimental research, two problems related to the control of are studied, namely:

1. The problem of the deterioration suffered by the strentgh of the concrete sample due to the cut needed to extract it and the degree of repreex the differ confidence supplied samples.

2. The verification of the loss of strength in elements which have sample and the subsequent filling of the resulting hole.

\section{zusammenfassung}

Beítrag zur Bewertung der Haltbarkeitsqualität des Strukturbetons an Hand von Herausgesch. nittenen Zeugen-Proben

J. L. Ramírez Ortiz, Prof. Dr. Industrie-

J. M. Bárcena Díaz, Industrieingenieur

In dieser Probenuntersuchung werden zwei Probleme, die sich auf die Bau hen, studiert:

1. Das Problem der Beschädigung, welche die Haltbarkeit der Betonprobe auf Grund des zur Entnahme notwen digen Schnittes erleidet. Der Erscheinungs und Vertraut. Der, Eruns die verschiedenen Durch

2. Nachprüfung des Haltbarkeitsverlustes der Elemente, die zur Entnahme wurden. Das entstanden Loch wurde hinterher wieder aufgefüllt. 